Journal of The Magnetics Society of Japan Vol. 13, Supplement, No. S1 (1989)

(C) 1989 by The Magnetics Society of Japan

\title{
CURVATURE OF PERPENDICULAR MAGNETIZATION TRANSITIONS ACROSS TRACK
}

\author{
James E. MONSON*, Kazuko OKUDA**, Richard E. FAYLING* $x^{*}$
}

Harvey Mudd College, Claremont, CA 91711 , USA
$\star * * \quad$ TRL, IBM Japan Ltd., 5-19, Sanbancho, Chiyoda-ku, Tokyo 102, Japan
$\star * * \quad 3 M, 3 M$ Center 260-2A-05, St. Paul, MN 55144, USA

Abstract-.. The nature of the perpendicular recording process at track edges is of interest in the design of very high track density recoriing systems. Recording experiments carried out on a large-scale model exhibit across-track curvature of the recorded transitions of perpendicular magnetization. Calculations using a simple three-dimensional analytical recording model suggest that the curvature is associated with the across-track variation of the demagnetizing field produced by the recorded magnetization pattern.

\section{INTRODUCTION}

A distinguishing feature of the perpendicular recording mode compared with the longitudinal mode is the rapid fall-off of the writing field at the track edges, first reported by Iwasaki and Nakamura[1]. This characteristic has important consequences for the design of very high track density recording systems, and a number of workers have reported on various aspects of narrow track perpendicular recording. Studies have utilized large-scale models[2], analytical models [3], and laboratory disk drives[4], producing increased understanding of the full, three-dimensional perpendicular recording process.

This paper reports on the phenomenon of across-track curvature of recorded transitions of perpendicular magnetization, which has been measured on a large-scale model and predicted from an analytical recording model.

\section{SCALE MODEL RESULTS}

In the large-scale model experiments, a probe head was used in the stand-still mode to record a region of reversed magnetization on a premagnetized barium ferrite medium mounted on a soft magnetic back layer. The barium ferrite mediun was then translated at constant velocity in contact with a conventionally sized ring head to determine the down track locations of the magnetization transitions on the leading and trailing edges of the reversed magnetization region. The ring head was then displaced incrementally across the medium and the measurement repeated to map the loci of the transitions.

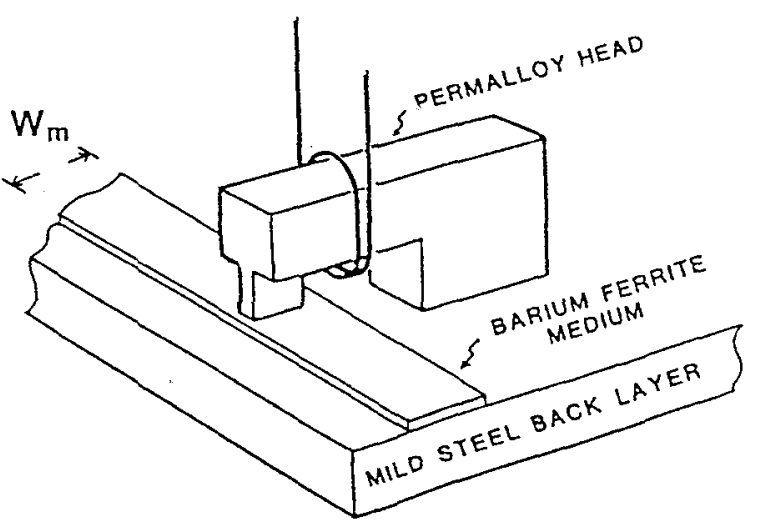

Fig. 1. Large-scale model apparatus.

The scale model perpendicular mediun was a commercially available flexible permanent magnet material fabricated of perpendicularly oriented barium ferrite particles suspended in a rubber binder. The mediun had a perpendicular coercivity of 2600 De, remanence of $1800 \mathrm{G}$, thickness 375 $\mu \mathrm{m}$, and width $12.6 \mathrm{~mm}$. Stand-still 
recording was done as follows. Initially, the medium was magnetized to its maximum remanent magnetization state in a large electromagnet. It was then placed on a high permeability mild steel plate which served as a back layer. The large-scale probe head was positioned in contact with the medium as illustrated in Fig. 1. The probe (main pole) had thickness $T_{\text {m }}-1 \mathrm{~mm}$ and width $W-$ $9.5 \mathrm{~mm}$. Both the main pole and core for flux return were fabricated from permalloy. A 1.5 A current was applied to the 100 turn coil to write a region of reversed magnetization.

\section{READ}

\section{SIGNAL ( $v$ )}

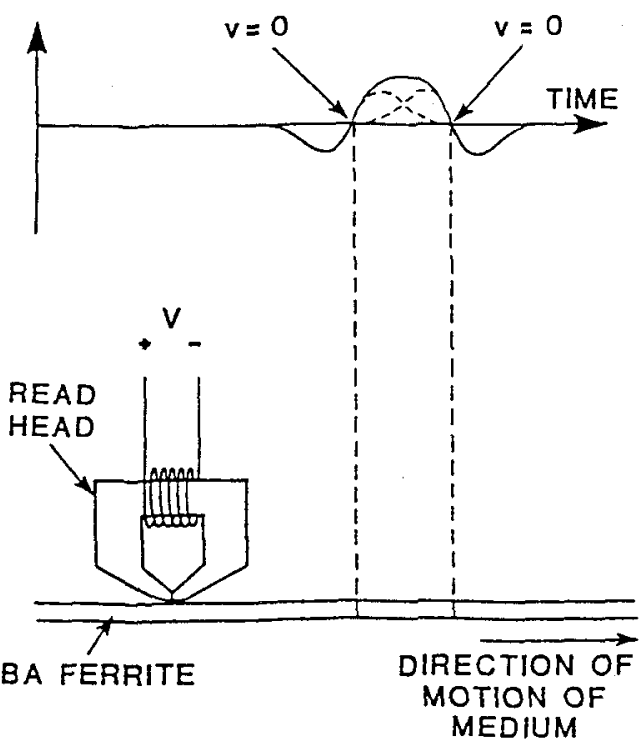

Fig. 2. Configuration for measuring transition locations.

After writing, the barium ferrite medium was removed and spliced to a strip of clear polyester film to form a continuous loop. A laboratory tape transport with vacuum tension control drove the loop in contact with a ring head at constant velocity. The ring head had gap length of $0.1 \mathrm{~mm}$ and track width of $0.5 \mathrm{~mm}$. The read head voltage had the shape indicated in Fig. 2 , which is the superposition of readback pulses from perpendicular magnetization transitions. The locations of the transitions correspond to the zero crossings of the readback voltage waveform. These locations are calculated from the waveform timing and the head-medium velocity.

The read head was repositioned laterally across the track width, and the voltage waveforms measured to generate transition locations as a function of distance $z$ from track center. Fig. 3 shows the loci of the leading and trailing edge transitions measured in this way. These curves indicate the boundaries of the reverse magnetization region recorded by the stationary probe head. The position of the recording pole tip is shown by dashed lines. Note that the transition loci show observable curvature, even for $z / W$ as low as 0.4. Fig. 3 exaggerates the curvature because of the difference in scales between $x$ and $z$ coordinates.

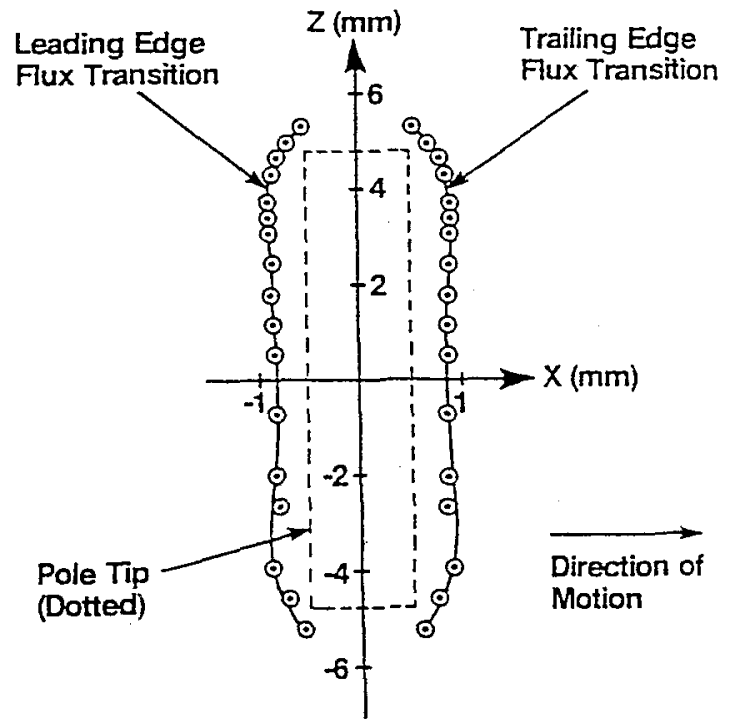

Fig. 3. Perpendicular magnetization transition pattern recorded with largescale model.

ANALYTICAL RECORDING MODEL

\section{Head field}

To aid in the understanding of the three-dimensional aspects of perpendicular recording, simple analytical models for head 
and medium were developed. The head field model generates the full 3-D head field for a single pole head over a soft magnetic back layer using the method presented by Okuda, et al. [5]. The method uses two submodels, each of which gives the field in its appropriate region. $F i g .4$ (a) shows the head geometry. For the region $|x|>T_{I} / 2+$ $S / 2$ or $|z|>W / 2+S / 2$, a technique using a suitably chosen superposition of Iindholm geometries[6] to satisfy the head and back layer potentials gives analytical field expressions. In the remaining region close to the head, a continuous charge distribution method which is a two-dimensional extension of the approximation of szczech et al.[7] also gives an analytical solution[8] for the field. The solutions for the two regions are matched at the boundary.

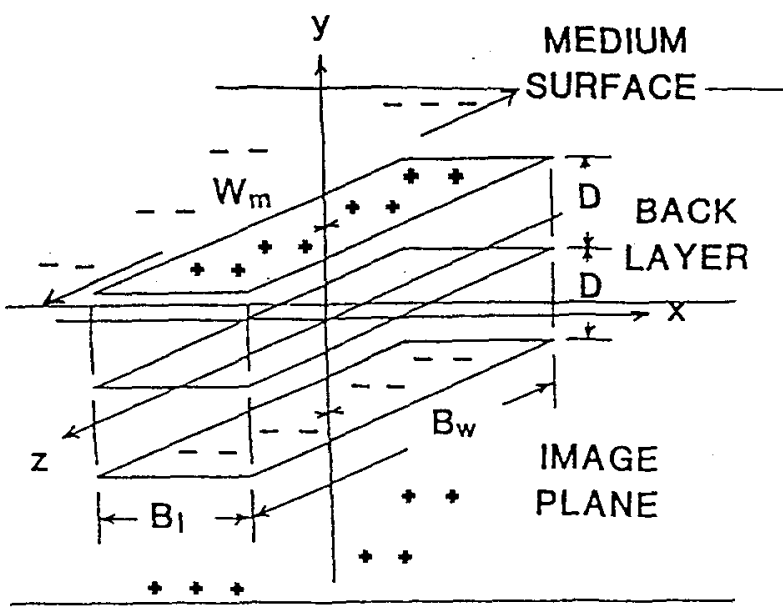

(b)

Fig. 4. (a) Head geometry. (b) Medium geometry: charge distribution for standstill recording.

\section{Medium}

In the model for the medium, it is assumed that the magnetization has only a perpendicular (y) component, and that the medium is magnetized to either positive or negative remanence as a function of $(x, z)$ and its magnetic history. Magnetization is assumed not to vary with $y$. These are reasonable starting assumptions for a medium in which the demagnetizing field for uniform magnetization is less than the coercivity, the condition which applies to the medium of the scale model.

In the stand-still recording mode where the uniformly magnetized medium has a region of reversed magnetization written on it, it is assumed that initially, the reversal region has a rectangular boundary. This enables the demagnetizing field to be readily calculated. Fig. 4 (b) shows the geometry of the written reversed region. The demagnetizing field can be determined from the surface charge on the top of the medium and its image in the back layer, which is assumed to be infinitely thick and permeable. By superposition, the $y$ component of the demagnetizing field is given by the demagnetizing field of a uniformly negatively magnetized medium, 
infinite in extent, minus the field from a rectangular charged sheet and its inage these charges corresponding to positive magnetization of twice the strength of the original negative magnetization. The $y$ component of demagnetizing field is given by:

$$
H_{d y}=4 \pi M_{I} H_{d b y}
$$

where,

$$
H_{d b y}(x, y, z)=H(x, y-D, z)-H(x, y+D, z)
$$

with

$$
\begin{aligned}
& H(x, y, z)=\frac{1}{4 \pi}\left(H_{+}^{(1)}\left(y, z+\frac{W_{m}}{2}\right)-H_{+}^{(1)}\left(y, z-\frac{W_{z}}{2}\right)\right. \\
& -H^{(1)}\left(y, z+\frac{W_{g 1}}{2}\right)+H^{(1)}\left(y, z-\frac{W_{m}}{2}\right) \\
& -2 H^{(2)}\left(x+\frac{B_{1}}{2}, y, z+\frac{B_{w}}{2}\right)+2 H^{(2)}\left(x-\frac{B_{1}}{2}, y, z+\frac{B_{w}}{2}\right) \\
& \left.+2 H^{(2)}\left(x+\frac{B_{1}}{2}, y, z-\frac{B_{w}}{2}\right)-2 H^{(2)}\left(x-\frac{B_{1}}{2}, y, z-\frac{B_{m}}{2}\right)\right) \\
& H_{ \pm}^{(1)}(y, z)= \pm \tan ^{-1} \frac{z}{y} \\
& H^{(2)}(x, y, z)=\tan ^{-1} \frac{z \cdot x}{y\left(x^{2}+y^{2}+z^{2}\right)^{1 / 2}}
\end{aligned}
$$

\section{Writing Process}

The writing process is assumed to occur as follows.

1. Magnetic transitions are symmetrical step transitions, i. e., the transition spreading parameter a is taken to be zero.

2. Only the $y$ components of fields are effective in the writing process, and writing is caused by the total field at the middle of the medium $(y-D / 2)$.

3. The $x$ dimension of the written rectangle is determined for $z-0$ by adjusting head field amplitude and transition location so that the slope $\mathrm{dH}_{\mathrm{hy}} / \mathrm{dx}=-\mathrm{dH}_{\mathrm{dy}} / \mathrm{dx}$ for $\mathrm{H}_{\mathrm{h}}-\mathrm{H}_{\mathrm{c}}$. The transition occurs at the $x$ for which these conditions are satisfied, because $H_{d y}(z=0)$ is zero for this value of $x$.

4. The $z$ dimension is chosen from the condition that $\mathrm{H}_{\mathrm{h}}=\mathrm{H}_{\mathrm{c}}$ for $\mathrm{x}=0$.
5. Transitions are written where the total field, head field plus demagnetizing field, is equal to the coercivity. The locus of this condition gives the boundary of the reversed magnetization region.

In this simple model, the write process stops at step 5. This serves as a first iteration. The next step would be to recalculate the demagnetizing field from the magnetization pattern found in step 5. and iterate step 5. until suitable convergence occurs. The demagnetizing field calculation could be done numerically or by a more refined approximation of the magnetization region by rectangular shapes.

\section{Example}

Figs. 5-7 show some results from a typical example using the analytical model described above. The model parameters are similar to those of the scale model experiment. Coercivity is $2700 \mathrm{Oe}$, remanence $1800 \mathrm{G}$, and the head field, $\mathrm{H}_{0}$, at the back layer in the center of the probe is $4600 \mathrm{Oe}$. As in the scale model, recording is done in contact $(S=D)$. A head field of 4600 oe for a coil current of 1.5 A in 100 turns is equivalent to a head efficiency of 908 , head efficiency taken as magneto-motive force across the medium divided by magneto-motive force applied to the coil.

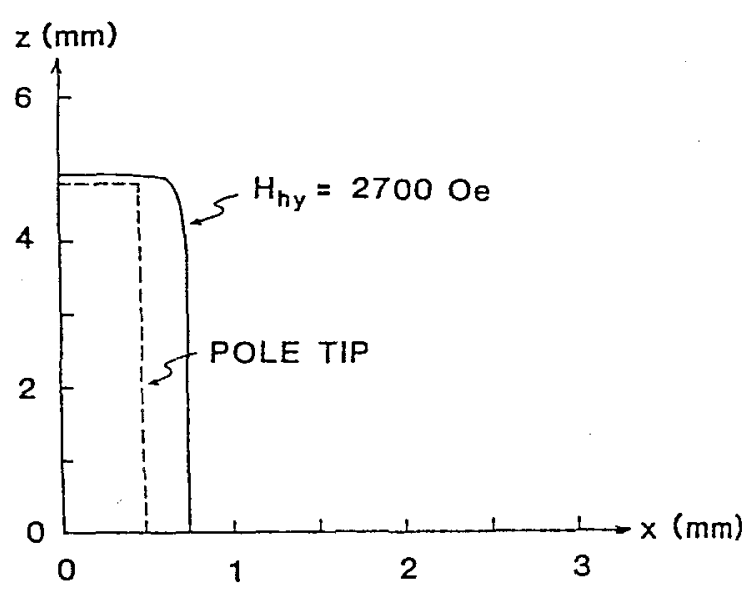

Fig. 5. Head field contour for $H_{\text {hy }}(x, z)=$ 2700 Oe for $y=0.2 \mathrm{~mm}$. 
Fig. 5 shows the contour of head field equal to 2700 De for $y=0.2 \mathrm{~mm}$, the middle of the medium. Note that there is no down track curvature for this contour.

The results of the model for the transition location at $z=0$ are illustrated in Fig. 6. For $\mathrm{H}_{0}-4600 \mathrm{Oe}$, the $\mathrm{x}$ location of the transition which matches $\mathrm{dH}_{\mathrm{hy}} / \mathrm{dx}-$ $-\mathrm{dH}_{\mathrm{dy}} / \mathrm{dx}$ for $\mathrm{H}_{\mathrm{h}}=2700$ Oe is $.78 \mathrm{~mm}$.

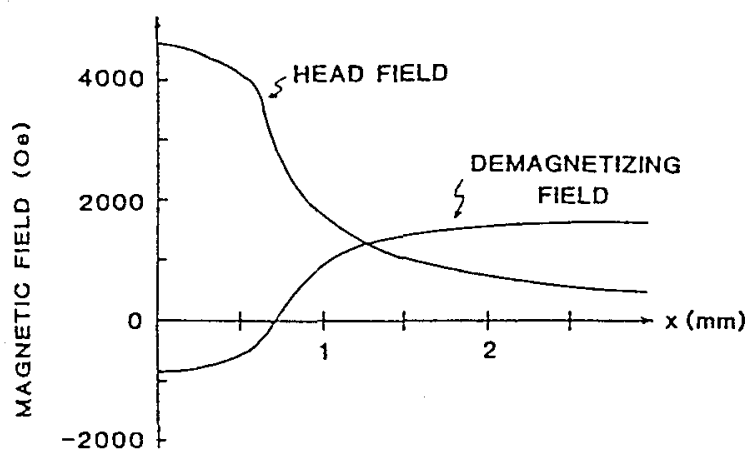

Fig. 6. y components of head field and medium's demagnetizing field as a function of $x$ for $z=0 . B_{1}=1.55 \mathrm{~mm}, B_{w}=10 \mathrm{~mm}$, $W_{m}=12.6 \mathrm{~mm}$, and $\mathrm{y}=0.2 \mathrm{~mm}$.

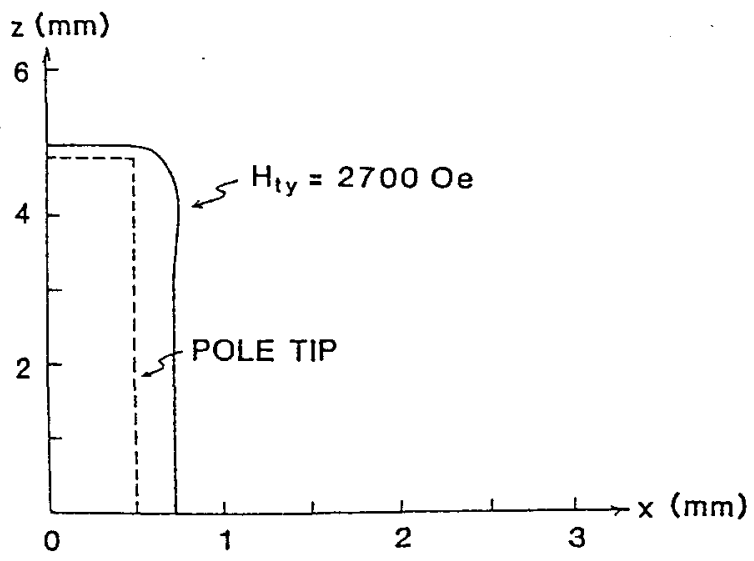

Fig. 7. Contour of $\mathrm{H}_{\mathrm{ty}}(\mathrm{x}, \mathrm{z})-2700$ oe for $y=0.2 \mathrm{~mm} . B_{1}=1.55 \mathrm{~mm}, B_{w}=10 \mathrm{~mm}$, and $\mathrm{W}_{\mathrm{m}}=12.6 \mathrm{~mm}$.
The writing of a reversed magnetization region is given by the condition that the total $\mathrm{y}$ field component, $\mathrm{H}_{\mathrm{ty}}$, which is the sum of the head field and the demagnetizing field is equal to $H_{c}, 2700$ oe in this example. Fig. 7 shows the contour for this condition, which is the boundary of the reversed magnetization region. Note the curvature down track for this contour.

\section{DISCUSSION}

The results presented above illustrate the curvature of perpendicular transitions measured on the large-scale model. A first approximation to the transition location, the contour of $y$ component of head field equal to coercivity, does not exhibit the measured curvature effect. The curvature appears related to the three-dimensional behavior of the demagnetizing fields from perpendicular magnetization transitions on finite tracks. The simple analytical model presented serves as a second approximation to the three-dimensional recording process.

Some effects not included in the model which might be important are variations in transition location through the thickness of the mediun, back layer effects[9] modifying the assumed charge representation of the medium, transition asymmetries, and transition spreading effects. Of these, transition asymnetries would be important to include for media with $4 \pi M_{I}>H_{c}$.

\section{CONCLUSION}

Curvature of perpendicular magnetization transitions has been shown, both experimentally and analytically. The results illustrate the richness of the full three-dimensional structure of perpendicular recording. These effects should be studied further for their implications in recording applications.

\section{REFERENCES}

[1] S. Iwasaki and Y. Nakamura, IEEE Trans. Magn., "The magnetic field distribution of a perpendicular recording head" Vol. MAG14, pp. 426-438, September 1978.

[2] K. Sueoka, F. Sai, and K. G. Ashar, IEEE Trans. Magn., "2-dimensional observation of 
recorded magnetization transitions in perpendicular recording" Vol. MAG-25, in press.

[3] J. E. Monson, IEEE Trans. Magn., "Fringing fields from step transitions of longitudinal and perpendicular magnetization on finite tracks" Vol. MAG- 24, pp. 3108-3110, September 1988.

[4] T. J. Beaulieu, D. J. Seagle, M. A. Meininger, and $C$. J. Spector, IEEE Trans. Magn., "Track density limitation for duallayer perpendicular recording in a rigid disk environment" Vol. MAG-25, in press.

[5] K. Okuda, K. Sueoka, and K. G. Ashar, IEEE Trans. Magn., "An analytical threedimensional field model of a perpendicular recording head" Vol. MAG-24, pp. 2479-2481, September 1988 .

[6] D. A. Lindholm, IEEE Trans. Magn., "Magnetic fields of finite track width heads" Vol. MAG-13, pp. 1460-1462, September 1977.

[7] T. J. Szczech, M. Steinbeck, and $M$. Jodeit, IEEE Trans. Magn., "Equations for the field components of a perpendicular magnetic head" Vol. MAG-18, pp. 229-232, January 1982 .

[8] K. Okuda, J. Mag. Soc. Japan, "3D head field analytical models of perpendicular recording head (II)" Vol. 13, pp. 109-112, 1989.

[9] Y. Nakamura and S. Iwasaki, Seminar of Applied Magnetics, Perpendicular Recording, pp. $7-16,1985$. 